\title{
BMJ Open Risk stratification for hydronephrosis in the evaluation of acute kidney injury: a cross-sectional analysis
}

\author{
Sri Lekha Tummalapalli (10 , ${ }^{1}$ John R. Zech, ${ }^{2}$ Hyung J. Cho, ${ }^{3,4}$ Celine Goetz ${ }^{5}$
}

To cite: Tummalapalli SL, Zech JR, Cho HJ, et al. Risk stratification for hydronephrosis in the evaluation of acute kidney injury: a crosssectional analysis. BMJ Open 2021;11:e046761. doi:10.1136/ bmjopen-2020-046761

- Prepublication history and additional supplemental material for this paper are available online. To view these files, please visit the journal online (http://dx.doi.org/10.1136/ bmjopen-2020-046761).

Received 09 November 2020 Accepted 29 July 2021

Check for updates

(C) Author(s) (or their employer(s)) 2021. Re-use permitted under CC BY-NC. No commercial re-use. See rights and permissions. Published by BMJ.

${ }^{1}$ Division of Healthcare Delivery Science and Innovation,

Department of Population Health Sciences, Weill Cornell Medicine, New York, New York, USA

${ }^{2}$ Department of Radiology, New York Presbyterian Hospital-

Columbia University Irving

Medical Center, New York, New

York, USA

${ }^{3}$ Chief Value Officer, New York City Health and Hospitals, New York, NY, USA

${ }^{4}$ Senior Fellow, Lown Institute, Brookline, MA, USA

${ }^{5}$ Director of Education,

Division of Hospital Medicine,

Department of Internal Medicine, Rush University Medical Center, Chicago, IL, USA

Correspondence to Dr Sri Lekha Tummalapalli; Ict4001@med.cornell.edu

\section{ABSTRACT}

Objective To validate an existing clinical decision support tool to risk-stratify patients with acute kidney injury (AKI) for hydronephrosis and compare the risk stratification framework with nephrology consultant recommendations. Setting Cross-sectional study of hospitalised adults with AKI who had a renal ultrasound (RUS) ordered at a large, tertiary, academic medical centre.

Participants Two hundred and eighty-one patients were included in the study cohort. Based on the risk stratification framework, $111(40 \%), 76(27 \%)$ and 94 $(33 \%)$ patients were in the high-risk, medium-risk and low-risk groups for hydronephrosis, respectively.

Outcomes Outcomes were the presence of unilateral or bilateral hydronephrosis on RUS.

Results Thirty-five patients (12\%) were found to have hydronephrosis. The high-risk group had $86 \%$ sensitivity and $67 \%$ specificity for identifying hydronephrosis. A nephrology consult was involved in $168(60 \%)$ patients and RUS was recommended by the nephrology service in $95(57 \%)$ cases. Among patients with a nephrology consultation, $9(56 \%)$ of the 16 total patients with hydronephrosis were recommended to obtain an RUS. Conclusions We further externally validated a risk stratification framework for hydronephrosis. Clinical decision support systems may be useful to supplement clinical judgement in the evaluation of AKI.

\section{INTRODUCTION}

Renal ultrasounds (RUS) are commonly ordered in hospitalised patients with acute kidney injury (AKI) to evaluate kidney size and parenchyma and to rule out obstructive pathology. ${ }^{12}$ Obstructive uropathy causing hydronephrosis is an uncommon cause of AKI, and clinical decision support systems (CDSS) could be used to inform which patients may benefit from RUS to rule out hydronephrosis. ${ }^{13-10}$

A risk stratification framework to identify patients who are at high risk of having hydronephrosis has been previously derived, but is currently underutilised. ${ }^{11}{ }^{12}$ Several factors may account for this, including lack of knowledge of the framework and lack of workflow integration. Recommendations from consulting nephrologists also inform

\section{Strengths and limitations of this study}

- Our study evaluates the utility of a clinical decision support tool to risk-stratify patients with acute kidney injury (AKI) for hydronephrosis.

- We found that the clinical decision support tool predicted hydronephrosis cases with high sensitivity and moderate specificity.

- Our results highlight that risk stratification frameworks may supplement clinical judgement in the evaluation of inpatient AKI.

- Limitations include the study being a single-centre study that evaluated only patients who had a renal ultrasound and not all patients with AKI.

- Our analysis was also limited to information present in the electronic health record.

RUS ordering for hospitalised patients with AKI; however, the relationship between risk stratification frameworks and nephrologist recommendations is unclear.

In this study, we further externally validated a risk stratification framework for hydronephrosis at our institution. We add to the literature by comparing the risk stratification tool with nephrology consultation recommendations for RUS ordering. We also examined the reason for the examination, incidental findings, and additional work-up from RUS. Understanding the potential role of CDSS to risk-stratify patients for hydronephrosis may improve the appropriateness of RUS ordering in the clinical evaluation of AKI. ${ }^{13}$

\section{METHODS}

\section{Study design and population}

We performed a cross-sectional study of hospitalised adults at an urban, 1134-bed academic medical centre in New York, New York. We included all patients who met the following three criteria: (1) admitted to the hospitalist service between 9 June 2013 and 24 October 2014; (2) experienced AKI (defined as creatinine rise $>0.3$ ); and (3) had a formal RUS 
Table 1 Baseline characteristics of patients by risk group for hydronephrosis

\begin{tabular}{|c|c|c|c|c|}
\hline & $\begin{array}{l}\text { High risk } \\
(\%, n=111)\end{array}$ & $\begin{array}{l}\text { Medium risk } \\
(\%, \mathrm{n}=76)\end{array}$ & $\begin{array}{l}\text { Low risk } \\
(\%, n=94)\end{array}$ & $P$ value \\
\hline \multicolumn{5}{|l|}{ Sociodemographics } \\
\hline \multicolumn{5}{|l|}{ Age } \\
\hline $18-39$ & 9 & 7 & 12 & \multirow[t]{4}{*}{0.30} \\
\hline $40-59$ & 19 & 22 & 31 & \\
\hline $60-74$ & 37 & 42 & 33 & \\
\hline$\geq 75$ & 35 & 29 & 24 & \\
\hline \multicolumn{5}{|l|}{ Sex } \\
\hline Male & 64 & 51 & 53 & \multirow[t]{2}{*}{0.15} \\
\hline Female & 36 & 49 & 47 & \\
\hline \multicolumn{5}{|l|}{ Race/ethnicity } \\
\hline Non-Hispanic white & 34 & 24 & 11 & \multirow[t]{4}{*}{$<0.001^{\star}$} \\
\hline Non-Hispanic black & 14 & 39 & 63 & \\
\hline Hispanic & 32 & 29 & 22 & \\
\hline Other & 20 & 8 & 4 & \\
\hline \multicolumn{5}{|l|}{ Risk criteria } \\
\hline History of hydronephrosis & 27 & 0 & 0 & $<0.001^{*}$ \\
\hline Non-black race & 86 & 61 & 37 & $<0.001^{*}$ \\
\hline History of recurrent UTIs & 18 & 3 & 1 & $<0.001^{*}$ \\
\hline $\begin{array}{l}\text { Diagnosis consistent with possible } \\
\text { obstruction }\end{array}$ & 70 & 45 & 16 & $<0.001^{*}$ \\
\hline Absence of nephrotoxins & 68 & 39 & 10 & $<0.001^{*}$ \\
\hline Absence of CHF & 92 & 86 & 44 & $<0.001^{*}$ \\
\hline Absence of prerenal AKI & 78 & 67 & 53 & $0.005^{\star}$ \\
\hline \multicolumn{5}{|l|}{ Laboratory results } \\
\hline Creatinine at admission, mean (SD) & $4.2(4.1)$ & $3.7(3.9)$ & $3.0(2.3)$ & $0.048^{*}$ \\
\hline Baseline creatinine, mean (SD) & $2(1.5)$ & $1.8(1.3)$ & $1.6(0.9)$ & 0.078 \\
\hline
\end{tabular}

$\chi^{2}$ tests were used to compare whether categorical variables differed by risk group for hydronephrosis. Analysis of variance tests were used to compare whether continuous variables (laboratory values) differed by risk group for hydronephrosis.

*Statistically significant at $\mathrm{p}<0.05$.

AKI, acute kidney injury; CHF, congestive heart failure; UTIs, urinary tract infections.

completed. Imaging was performed by trained, experienced ultrasonographers.

\section{Risk stratification}

Our primary predictor was high-risk, medium-risk, or lowrisk category for hydronephrosis based on the risk stratification framework developed by Licurse et al (online supplemental table 1). ${ }^{12}$ The following are the seven criteria included in the framework: a history of hydronephrosis (4 points), and non-black race, history of recurrent urinary tract infections (UTIs), diagnosis consistent with possible obstruction (abdominal or pelvic mass, benign prostatic hypertrophy, pelvic surgery, or neurogenic bladder), absence of exposure to inpatient nephrotoxic agents (aspirin $>81 \mathrm{mg}$, diuretics, ACE inhibitors or vancomycin), absence of congestive heart failure, and/or absence of prerenal AKI (pressor use or sepsis) (1 point each). A total score of 4 or more points was classified as high risk, 3 points as medium risk, and 2 or fewer points as low risk. ${ }^{12}$ We performed an additional sensitivity analysis defining prerenal AKI as pressor use, sepsis or history of hypotension, defined as at least two consecutive blood pressure measurements below $80 \mathrm{~mm} \mathrm{Hg}$ systolic or below $60 \mathrm{~mm} \mathrm{Hg}$ diastolic. Additional predictors were presence of nephrology consultation and RUS recommended by nephrology.

We performed a retrospective chart review of data in the electronic health record (EHR) to determine the presence or absence of each predictor, using only data available before the RUS was ordered. One author (JZ) performed the initial chart review, which was recorded in the REDCap web-based application (Vanderbilt University). Another author (CG) independently reviewed a random selection of $10 \%$ of medical records, with $96 \%$ agreement (Cohen's $\kappa=0.913, \mathrm{p}<0.001$ ). 
Table 2 Risk stratification versus nephrology consult recommendation for RUS in hospitalised patients with AKI and prevalence of hydronephrosis

\begin{tabular}{lccc}
\hline & $\begin{array}{l}\text { Total } \\
\text { cases } \\
(\mathbf{n = 2 8 1 )})\end{array}$ & $\begin{array}{l}\text { Hydronephrosis } \\
(\mathbf{n = 3 5 )}\end{array}$ & $\begin{array}{l}\text { Hydronephrosis } \\
(\mathbf{\%})\end{array}$ \\
\hline Risk stratification & & & \\
\hline High-risk & 111 & 30 & 27 \\
\hline Medium-risk & 76 & 1 & 1 \\
\hline Low-risk & 94 & 4 & 4 \\
\hline Nephrology consult recommendation & \\
\hline RUS recommended & 95 & 9 & 9 \\
\hline Not recommended & 73 & 7 & 10 \\
\hline $\begin{array}{l}\text { No nephrology } \\
\text { consult }\end{array}$ & 113 & 19 & 17 \\
\hline
\end{tabular}

AKI, acute kidney injury; RUS, renal ultrasound.

\section{Outcomes}

Our primary outcome was the presence of unilateral or bilateral hydronephrosis. Severity of hydronephrosis (mild, moderate and severe) was ascertained by radiologists in the official RUS report. Secondary outcomes were incidental findings in the RUS radiology report, urological procedure after RUS, and further imaging as a result of RUS during the hospitalisation. Incidental findings were classified into the following categories: increased echogenicity, simple cysts, complex cysts, renal atrophy or cortical thinning, non-obstructive nephrolithiasis, renal enlargement, renal mass, absence of kidney, obstructive nephrolithiasis, or other. ${ }^{14}$ We also determined the reason for RUS ordering based on comments written in the 'Reason for exam' field in the RUS order.

\section{Statistical analysis}

We described patient characteristics according to risk group for hydronephrosis, presence of nephrology consultation, and RUS recommended by the consult service. We determined the prevalence of patients with hydronephrosis by risk group, nephrology consultation, and RUS recommendation. $\chi^{2}$ and analysis of variance tests were performed to

\begin{tabular}{|c|c|c|c|}
\hline & $\begin{array}{l}\text { Hydronephrosis } \\
(\mathrm{n}=35)\end{array}$ & $\begin{array}{l}\text { No hydronephrosis } \\
(n=246)\end{array}$ & Total \\
\hline \multicolumn{4}{|l|}{ (A) High cut-off } \\
\hline High-risk & 30 & 81 & 111 \\
\hline $\begin{array}{l}\text { Medium-risk or } \\
\text { low-risk }\end{array}$ & 5 & 165 & 170 \\
\hline Total & 35 & 246 & 281 \\
\hline \multicolumn{4}{|l|}{ (B) Medium cut-off } \\
\hline $\begin{array}{l}\text { High-risk or } \\
\text { medium-risk }\end{array}$ & 31 & 156 & 187 \\
\hline Low-risk & 4 & 90 & 94 \\
\hline Total & 35 & 246 & 281 \\
\hline
\end{tabular}

Table 4 Factors significantly related to hydronephrosis in the study cohort

\begin{tabular}{lccl}
\hline Factor & Count & \multicolumn{1}{l}{ r } & $\begin{array}{l}\text { P value of Pearson } \\
\text { correlation }\end{array}$ \\
\hline History of hydronephrosis & 30 & 0.5 & $<0.001^{*}$ \\
\hline Recurrent UTI & 23 & 0.32 & $<0.001^{*}$ \\
$\begin{array}{l}\text { Diagnosis consistent with } \\
\text { obstruction }\end{array}$ & 127 & 0.2 & $<0.001^{*}$ \\
\hline $\begin{array}{l}\text { Non-black race } \\
\text { Absence of exposure to } \\
\text { nephrotoxic agents }\end{array}$ & 176 & 0.03 & 0.66 \\
\hline $\begin{array}{l}\text { Absence of CHF } \\
\text { Absence of prerenal AKI }\end{array}$ & 114 & 0.15 & $0.012^{*}$ \\
\hline
\end{tabular}

Pearson correlation coefficients were used to assess the correlation of factors in the risk score with hydronephrosis in our population.

*Statistically significant at $\mathrm{p}<0.05$.

AKI, acute kidney injury; CHF, congestive heart failure; UTI, urinary tract infections.

compare patient characteristics by risk group for hydronephrosis and nephrology consultation status. We reported the test characteristics of the risk stratification framework for identifying hydronephrosis. We calculated Pearson correlation coefficients to assess the correlation of factors in the risk score with hydronephrosis in our population. Methods to account for multiple comparisons, such as Bonferroni correction, were not employed. Analyses were performed in Stata V.15 statistical software. ${ }^{15}$

\section{Patient and public involvement}

Patients were not involved in the design of the study, including the research question, outcome measures, or conduct. Results will be disseminated to patient groups via email listservs and via publication in the peer-reviewed literature.

\section{RESULTS}

\section{Patient demographics}

Three hundred and twenty-two patients admitted to the hospitalist service received an RUS during the study period. Forty-one of these patients were not included because they did not have AKI. The final cohort included 281 patients. The mean age was 64 years, $57 \%$ of patients were male, and $63 \%$ were non-black (table 1 ).

\section{Risk stratification framework}

There were 111 (40\%), 76 (27\%), and 94 (33\%) patients in the high-risk, medium-risk, and low-risk groups for hydronephrosis, respectively. Patients in the high-risk group were less likely to be black ( $14 \%$ vs $63 \%, \mathrm{p}<0.001)$ and had higher creatinine levels at admission (4.2 mg/ $\mathrm{dL}$ vs $3.0 \mathrm{mg} / \mathrm{dL}, \mathrm{p}=0.048$ ) compared with those in the low-risk group (table 1). Thirty-five patients (12\%) were found to have hydronephrosis, of whom $30(86 \%)$ were captured in the high-risk group (table 2). Thirty of the high-risk patients $(27 \%)$, one medium-risk patient $(1 \%)$, and four of the low-risk patients $(4 \%)$ were found to have 
hydronephrosis on RUS. The high-risk group had $86 \%$ sensitivity and $67 \%$ specificity for identifying hydronephrosis (table 3A). The medium-to-high-risk group had $89 \%$ sensitivity and $37 \%$ specificity for identifying hydronephrosis (table 3). Among patients with hydronephrosis, 17 of 35 (49\%) had unilateral hydronephrosis and 18 of 35 (51\%) had bilateral hydronephrosis. Test characteristics did not meaningfully change after sensitivity analysis changing the definition of prerenal AKI (online supplemental table 2). Five of the seven criteria were found to be statistically significantly correlated with increased risk of hydronephrosis in our population (table 4).

\section{Nephrology consultation}

A nephrology consult was involved in 168 patients (60\%). Patients with nephrology consultation had fewer recurrent UTIs ( $2 \%$ vs $17 \%, \mathrm{p}<0.001)$, higher creatinine levels at admission $(4.7 \mathrm{mg} / \mathrm{dL}$ vs $2.2 \mathrm{mg} / \mathrm{dL}, \mathrm{p}<0.001)$, and higher baseline creatinine $(2.1 \mathrm{mg} / \mathrm{dL}$ vs $1.3 \mathrm{mg} /$ $\mathrm{dL}, \mathrm{p}<0.001)$ compared with those who did not have nephrology consultation (table 5). RUS was recommended by the nephrology service in 95 of 168 cases (57\%). Similarly, in the low-risk patient group, of the 61 low-risk patients $(65 \%)$ who had a nephrology consult, 35 $(57 \%)$ were recommended to obtain an RUS. Of the 95 patients recommended for an RUS, 9 (9\%) had hydronephrosis. Among patients with a nephrology consultation, $9(56 \%)$ of the 16 total patients with hydronephrosis were recommended to obtain an RUS (table 2).

Reason for examination, incidental findings and additional work-up

The majority of RUS were ordered for AKI or renal failure $(57 \%)$ or to rule out hydronephrosis or obstruction

Table 5 Baseline characteristics of patients by nephrology consultation

\begin{tabular}{|c|c|c|c|c|}
\hline & $\begin{array}{l}\text { Nephrology consult, } \\
\text { RUS recommended } \\
(\%, n=95)\end{array}$ & $\begin{array}{l}\text { Nephrology consult, } \\
\text { RUS not recommended } \\
(\%, n=73)\end{array}$ & $\begin{array}{l}\text { No nephrology } \\
\text { consult (\%, } \\
n=113)\end{array}$ & $P$ value \\
\hline \multicolumn{5}{|l|}{ Sociodemographics } \\
\hline \multicolumn{5}{|l|}{ Age } \\
\hline 18-39 & 12 & 7 & 9 & \multirow[t]{4}{*}{0.70} \\
\hline $40-59$ & 23 & 26 & 23 & \\
\hline $60-74$ & 41 & 37 & 34 & \\
\hline$\geq 75$ & 24 & 30 & 35 & \\
\hline \multicolumn{5}{|l|}{ Sex } \\
\hline Male & 56 & 56 & 58 & \multirow[t]{2}{*}{0.92} \\
\hline Female & 44 & 44 & 42 & \\
\hline \multicolumn{5}{|l|}{ Race/ethnicity } \\
\hline Non-Hispanic white & 28 & 22 & 20 & \multirow[t]{4}{*}{0.30} \\
\hline Non-Hispanic black & 31 & 36 & 44 & \\
\hline Hispanic & 28 & 34 & 23 & \\
\hline Other & 13 & 8 & 12 & \\
\hline
\end{tabular}

Risk criteria

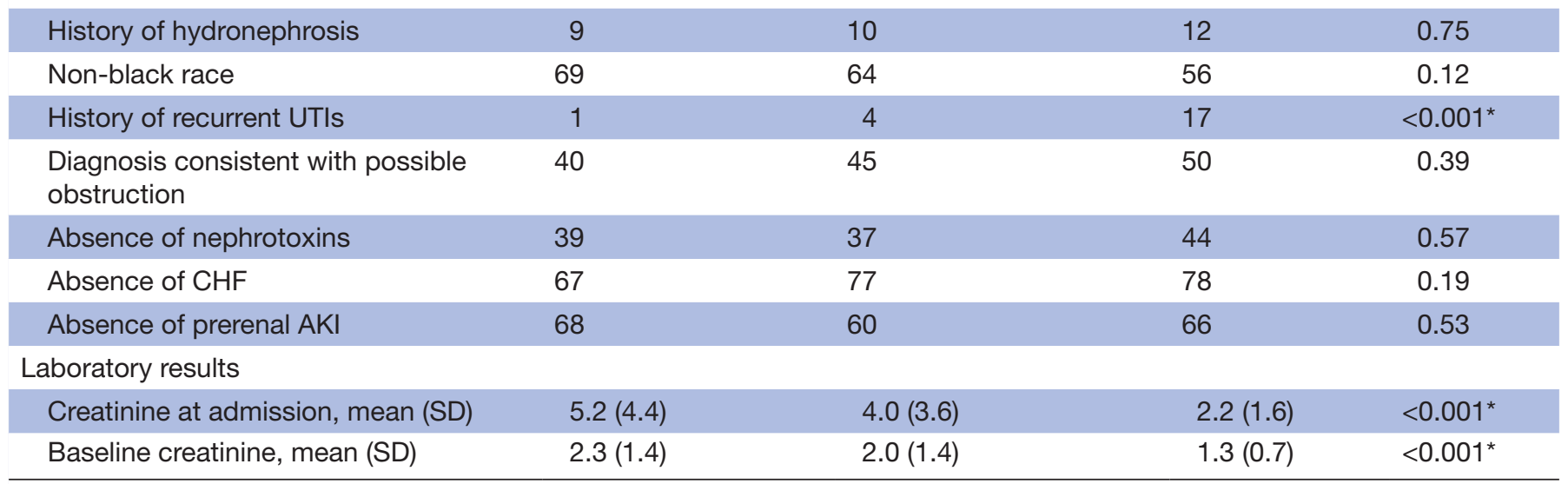

$\chi^{2}$ tests were used to compare whether categorical variables differed by nephrology consultation status. Analysis of variance tests were used to compare whether continuous variables (laboratory values) differed by nephrology consultation status.

*Statistically significant at $\mathrm{p}<0.05$.

AKI, acute kidney injury; CHF, congestive heart failure; RUS, renal ultrasound; UTIs, urinary tract infections. 
Table 6 Reason for RUS examination

Reason for examination

Total

$(\mathrm{n}=236)^{*}$, n (\%)

AKI or renal failure

$134(57)$

Rule out hydronephrosis or obstruction

$56(24)$

Oliguria or anuria

9 (4)

Rule out pyelonephritis or abscess

$6(3)$

Other

6 (3)

Evaluate for nephrolithiasis

4 (2)

ESRD or new ESRD

4 (2)

CKD

4 (2)

Recurrent UTIs

$3(1)$

Costovertebral tenderness or flank pain

$3(1)$

Evaluate ureteral stent or nephrostomy tube

$2(1)$

Haematuria

$2(1)$

Concern for hepatorenal syndrome

$2(1)$

Renal cyst or mass seen on CT scan

$1(0.4)$

Obstructive nephrolithiasis

$1(0.4)$

*Excludes examinations with 'Reason for exam' listed as blank or N/A.

AKI, acute kidney injury; CKD, chronic kidney disease; ESRD, endstage renal disease; N/A, not applicable; RUS, Renal ultrasound; UTIs, urinary tract infections.

(24\%). Additional reasons for examination were AKI indicated predominantly by oliguria or anuria, rule out pyelonephritis or abscess, evaluate for nephrolithiasis, and other (table 6). Twenty-four percent of RUS listed rule out hydronephrosis or obstruction as the reason for examination. Only three RUS specifically listed the reason for examination as evaluating kidney size, echogenicity, or chronicity of kidney disease.

Only 37 patients (13\%) had no abnormal findings on RUS. Even within the low-risk group, only $16 \%$ of RUS were without abnormalities. The most common finding was increased echogenicity, which occurred in 195 patients $(69 \%)$. One hundred and two (36\%) had simple cysts and 34 (12\%) had complex cysts (table 7).

Four patients $(1.4 \%)$ in the entire cohort underwent a urological procedure, all of whom were in the highrisk group. Thirty-five patients (12\%) received further imaging as a result of the RUS findings. Of the patients who underwent further imaging, $21(60 \%), 11(31 \%)$ and $3(9 \%)$ were in the high-risk, medium-risk and low-risk groups, respectively.

\section{DISCUSSION}

We found that the high-risk group in a risk stratification framework identified $86 \%$ of episodes of hydronephrosis in hospitalised patients with AKI who obtained an RUS. ${ }^{11} 12$ The majority (60\%) of RUS were ordered on medium-risk or low-risk patients, while only $3 \%$ of these patients were found to have hydronephrosis. Our findings highlight the potential additive role of CDSS for hydronephrosis in supplementing clinical judgement in the evaluation of AKI.

Prior analyses ${ }^{41}$ have externally validated the Licurse et $a l^{12}$ risk stratification framework. Ip et $a l^{11}$ found $93.4 \%$ sensitivity and $25.1 \%$ specificity for hydronephrosis, similar to our results, using a cut-off between low-risk

Table 7 Ultrasound findings by risk group for hydronephrosis

\begin{tabular}{|c|c|c|c|c|}
\hline Finding & $\begin{array}{l}\text { High-risk } \\
(\%, n=111)\end{array}$ & $\begin{array}{l}\text { Medium-risk } \\
(\%, n=76)\end{array}$ & $\begin{array}{l}\text { Low-risk } \\
(\%, n=94)\end{array}$ & $P$ value \\
\hline Increased renal parenchymal echogenicity & 65 & 72 & 72 & 0.41 \\
\hline Other & 25 & 20 & 27 & 0.55 \\
\hline Complex cyst & 14 & 12 & 11 & 0.82 \\
\hline Renal atrophy or cortical thinning & 11 & 13 & 7 & 0.47 \\
\hline No abnormalities & 10 & 15 & 16 & 0.41 \\
\hline Non-obstructive nephrolithiasis & 8 & 5 & 9 & 0.69 \\
\hline Renal mass & 4 & 3 & 2 & 0.81 \\
\hline
\end{tabular}

There were no patients with staghorn calculi, anatomical urinary tract abnormalities, mass of the genitourinary tract, other abdominal mass, pelvic kidney or horseshoe kidney.

$\chi^{2}$ tests were used to compare whether variables differed by risk group for hydronephrosis.

${ }^{*}$ Statistically significant at $\mathrm{p}<0.05$. 
and medium-risk/high-risk patients. ${ }^{4}$ Our results support the use of a higher cut-off between low-risk/medium-risk and high-risk patients to improve specificity with minimal reduction in sensitivity.

Given that this risk stratification framework has been externally validated and has promising test characteristics, the next step is to prospectively implement and evaluate it. The American College of Radiology supports the use of CDSS as a method to ensure appropriate imaging, and CDSS has been associated with decreased imaging utilisation. ${ }^{1617}$ A pragmatic randomised trial could assess if the risk stratification framework built within an EHRenabled CDSS versus usual care improves AKI diagnosis, management, and/or appropriateness of imaging.

The risk stratification framework studied was aimed specifically at identifying obstructive pathology on RUS in patients with AKI. To assess if consulting or primary teams requesting RUS were evaluating factors other than hydronephrosis, such as size or echogenicity of kidneys, we assessed the reasons for RUS ordering listed in the 'Reason for exam' field. The majority of RUS were ordered for AKI or renal failure (57\%), without further explanation. We were thus unable to reliably assess the clinical rationale for RUS ordering.

We found that the majority $(87 \%)$ of RUS had incidental findings, which was similar to prior estimates. ${ }^{18}$ Incidental findings pose challenges to physicians and patients, and can cause patient anxiety and additional interventions. ${ }^{19}$ Importantly, over $10 \%$ of patients had important incidental findings, such as complex cysts, which may require further imaging and evaluation. The high prevalence of incidental findings with RUS imaging raises questions about when disclosure of these findings is warranted. ${ }^{20}$ While the majority of RUS had abnormalities, there was a low prevalence of urological procedures and further imaging during the hospitalisation.

The major strength in our approach was the detailed chart review used in our analysis. There are also limitations to our results. We conducted a single-site study and did not assess all patients with AKI, only those who had an RUS ordered. Our analysis was limited to information present in the EHR, so we did not capture nephrology consult recommendations that may have been given verbally. In some cases where RUS was obtained prior to nephrology consultation, it was unclear based on the available documentation whether the consultant would have also recommended the study. Lastly, the definition used for prerenal AKI was use of pressors or history of sepsis, which may lack sensitivity for other clinically significant hypovolaemic states. ${ }^{12}$

In conclusion, we further validated a risk stratification framework for hydronephrosis at our institution. There was a very high prevalence of incidental findings on RUS, but additional imaging and procedures were low during the inpatient hospitalisation. Our findings highlight the potential utility of CDSS to supplement comprehensive clinical evaluation in AKI.
Twitter Sri Lekha Tummalapalli @LTummalapalli

Acknowledgements The authors thank Dr Andrew S Dunn for editorial assistance.

Contributors Research idea and study design: SLT, JZ, HC, CG. Data acquisition: JZ, CG. Data analysis/interpretation: SLT, JZ. Statistical analysis: SLT, JZ. Supervision or mentorship: HC, CG. All authors contributed important intellectual content during manuscript drafting and revision and approved the final manuscript.

Funding The authors have not declared a specific grant for this research from any funding agency in the public, commercial or not-for-profit sectors.

Competing interests SLT receives consulting fees from Bayer, outside of the submitted work.

Patient consent for publication Not required.

Ethics approval The Mount Sinai Institutional Review Board reviewed and approved the design of the present study.

Provenance and peer review Not commissioned; externally peer reviewed.

Data availability statement Data are available upon reasonable request. Data are available from the authors upon reasonable request.

Supplemental material This content has been supplied by the author(s). It has not been vetted by BMJ Publishing Group Limited (BMJ) and may not have been peer-reviewed. Any opinions or recommendations discussed are solely those of the author(s) and are not endorsed by BMJ. BMJ disclaims all liability and responsibility arising from any reliance placed on the content. Where the content includes any translated material, BMJ does not warrant the accuracy and reliability of the translations (including but not limited to local regulations, clinical guidelines, terminology, drug names and drug dosages), and is not responsible for any error and/or omissions arising from translation and adaptation or otherwise.

Open access This is an open access article distributed in accordance with the Creative Commons Attribution Non Commercial (CC BY-NC 4.0) license, which permits others to distribute, remix, adapt, build upon this work non-commercially, and license their derivative works on different terms, provided the original work is properly cited, appropriate credit is given, any changes made indicated, and the use is non-commercial. See: http://creativecommons.org/licenses/by-nc/4.0/.

ORCID iD

Sri Lekha Tummalapalli http://orcid.org/0000-0002-6513-8460

\section{REFERENCES}

1 Waikar SS, Liu KD, Chertow GM. Diagnosis, epidemiology and outcomes of acute kidney injury. Clin J Am Soc Nephrol 2008;3:844-61.

2 Chertow GM, Burdick E, Honour M, et al. Acute kidney injury, mortality, length of stay, and costs in hospitalized patients. J Am Soc Nephrol 2005;16:3365-70.

3 Young A, Crawford T, Pierre AS, et al. Renal ultrasound provides low utility in evaluating cardiac surgery associated acute kidney injury. $J$ Cardiothorac Surg 2017;12:75.

4 Leaf DE, Srivastava A, Zeng X, et al. Excessive diagnostic testing in acute kidney injury. BMC Nephrol 2016;17:9.

5 Stuck KJ, White GM, Granke DS, et al. Urinary obstruction in azotemic patients: detection by sonography. AJR Am J Roentgenol 1987;149:1191-3.

6 Ritchie WW, Vick CW, Glocheski SK, et al. Evaluation of azotemic patients: diagnostic yield of initial us examination. Radiology 1988;167:245-7.

7 Podoll A, Walther C, Finkel K. Clinical utility of gray scale renal ultrasound in acute kidney injury. BMC Nephrol 2013;14:188.

8 Remer EM, Papanicolaou N, Casalino DD, et al. ACR Appropriateness Criteria $(\mathbb{R})$ on renal failure. Am J Med 2014;127:1041-8.

9 Gottlieb RH, Weinberg EP, Rubens DJ, et al. Renal sonography: can it be used more selectively in the setting of an elevated serum creatinine level? Am J Kidney Dis 1997;29:362-7.

10 Liu KD, Chertow GM. Curbing the use of ultrasonography in the diagnosis of acute kidney injury: Penny wise or pound foolish?: Comment on "Renal ultrasonography in the evaluation of acute kidney injury". Arch Intern Med 2010;170:1907-8.

11 Ip IK, Silveira PC, Alper EC, et al. External validation of risk stratification strategy in the use of renal ultrasonography in the evaluation of acute kidney injury. J Hosp Med 2016;11:763-7.

12 Licurse A, Kim MC, Dziura J, et al. Renal ultrasonography in the evaluation of acute kidney injury: developing a risk stratification framework. Arch Intern Med 2010;170:1900-7. 
13 Durand DJ, Lewin JS, Berkowitz SA. Medical-Imaging stewardship in the accountable care era. N Engl J Med 2015;373:1691-3.

14 Mazziotti S, Cicero G, D'Angelo T, et al. Imaging and management of incidental renal lesions. Biomed Res Int 2017;2017:1854027.

15 StataCorp. Stata statistical software: release 15. College Station, TX: StataCorp LLC, 2017.

16 Association ACoR-RBM. Best practices guidelines on imaging clinical decision support systems. Available: https://www.acr.org/-/ media/ACR/Files/Clinical-Resources/Clinical-Decision-Support/Best Practices for Imaging CDS Systems1.pdf [Accessed 1 Jun 2021].

17 Sistrom CL, Dang PA, Weilburg JB, et al. Effect of computerized order entry with integrated decision support on the growth of outpatient procedure volumes: seven-year time series analysis. Radiology 2009;251:147-55

18 Gamss R, Stein MW, Rispoli JM, et al. What is the appropriate use of renal sonography in an inner-city population with new-onset acute kidney injury? J Ultrasound Med 2015;34:1639-44.

19 Lumbreras B, Donat L, Hernández-Aguado I. Incidental findings in imaging diagnostic tests: a systematic review. Br J Radiol 2010;83:276-89.

20 Bunnik EM, van Bodegom L, Pinxten W, et al. Ethical framework for the detection, management and communication of incidental findings in imaging studies, building on an interview study of researchers' practices and perspectives. BMC Med Ethics 2017;18:10. 